\title{
Quercetin/ $\beta$-cyclodextrin inclusion complex embedded nanofibres: Slow release and high solubility
}

\author{
Zeynep Aytac ${ }^{\mathrm{a}, \mathrm{b}}$, Semran Ipek Kusku ${ }^{\mathrm{b}, \mathrm{c}}$, Engin Durgun ${ }^{\mathrm{a}, \mathrm{b}}$, Tamer Uyar ${ }^{\mathrm{a}, \mathrm{b}, *}$ \\ a Institute of Materials Science \& Nanotechnology, Bilkent University, Ankara 06800, Turkey \\ ${ }^{\mathrm{b}}$ UNAM-National Nanotechnology Research Center, Bilkent University, Ankara 06800, Turkey \\ ' Department of Engineering Physics, Istanbul Medeniyet University, 34700 Göztepe-Istanbul, Turkey
}

\section{A R T I C L E I N F O}

\section{Article history:}

Received 6 July 2015

Received in revised form 30 October 2015

Accepted 11 November 2015

Available online 12 November 2015

\section{Keywords:}

Electrospinning

Nanofibres

Polyacrylic acid

Quercetin

$\beta$-Cyclodextrin

Modelling

Phase solubility

Antioxidant activity

Photostability

\begin{abstract}
A B S T R A C T
Electrospinning of polyacrylic acid (PAA) nanofibres (NF) incorporating $\beta$-cyclodextrin inclusion complex $(\beta-C D-I C)$ of quercetin $(Q U)$ was performed. Here, $\beta-C D$ was used as not only the crosslinking agent for PAA nanofibres but also as a host molecule for inclusion of QU. The phase solubility test showed enhanced solubility of QU due to the inclusion complexation; in addition, the stoichiometry of $\mathrm{QU} / \beta-\mathrm{CD}-\mathrm{IC}$ was determined to be $1: 1$. Computational modelling studies confirmed that $1: 1$ and $1: 2$ complex formation are desirable; $1: 1$ complex formation was chosen to have higher weight loading of QU. SEM images showed that PAA/QU/ $\beta$-CD-IC-NF were bead-free and uniform. XRD indicated that $\mathrm{PAA} / \mathrm{QU} / \beta-\mathrm{CD}-\mathrm{IC}-\mathrm{NF}$ were amorphous in nature without the crystalline peaks of QU. Comparative results revealed that the release profile of $\mathrm{QU}$ from $\mathrm{PAA} / \mathrm{QU} / \beta-\mathrm{CD}-\mathrm{IC}-\mathrm{NF}$ was much slower but greater in total than from PAA/QU/ $\beta$-CD-IC-film. Moreover, high antioxidant activity and photostability of QU was achieved in PAA/QU/ $\beta$-CD-IC-NF.
\end{abstract}

(c) 2015 Elsevier Ltd. All rights reserved.

\section{Introduction}

Nanofibres have been used in filtration, catalysis, packaging, medical and energy applications, owing to their unique properties like high surface area-to-volume ratio and highly porous structure (Bhardwaj \& Kundu, 2010). One of the most convenient and appropriate methods to produce nanofibres is electrospinning, due to its efficiency and ability to produce nanofibres from a variety of materials, such as polymers, inorganic materials and composites (Bhardwaj \& Kundu, 2010). In addition, nanofibres can be functionalised with different compounds including drugs, antioxidant and antibacterial agents, flavours and fragrances (Agarwal, Wendorff, \& Greiner, 2008; Bhardwaj \& Kundu, 2010).

Quercetin (QU) (Fig. 1a) has been ubiquitously used in the food and pharmaceutical industries, due to its exceptional antiinflammatory, anti-allergy, antioxidant and antitumour activities. In nature, it has been found in onion, apple, green and black tea (Mercader-Ros et al., 2012). The usage of this molecule is restricted by its poor water solubility, low oral bioavailability and fast

\footnotetext{
* Corresponding author at: Institute of Materials Science \& Nanotechnology, Bilkent University, Ankara 06800, Turkey.

E-mail address: tamer@unam.bilkent.edu.tr (T. Uyar).
}

photodegradation. Therefore, developing a new carrier system is of vital importance for QU to be used in many application areas. Some studies related with the usage of electrospun nanofibres as a carrier for QU exist in the literature (Li, Wang, \& Yu, 2014; Xing et al., 2012).

Inclusion complexation of poorly soluble compounds with cyclodextrins (CDs) (Fig. 1b) is a well-known encapsulation approach. Since complexation is an effective method not only for improving the solubility and bioavailability but also for protecting compounds and enhancing their stability against oxygen, temperature and light (Del Valle, 2004). CDs are $\alpha$-1,4-linked cyclic oligosaccharides of D-glucopyranose units that have truncated cone structure (Fig. 1c). Native CDs having 6,7 and 8 glucopyranose units are named as $\alpha-C D, \beta-C D$ and $\gamma-C D$, respectively. CDs presents a hydrophilic exterior that makes them water soluble whereas the hydrophobic cavity of CDs enables the formation of inclusion complexes (ICs) with hydrophobic compounds. The polar-apolar interaction between the polar water molecules existing in the CD cavity and the apolar wall of the cavity induces an energetically unfavourable state. The substitution of highenthalpy water molecules with a guest molecule results in a cyclodextrin inclusion complex (CD-IC) formation between host (CD) and guest molecule. Most of the applications including food 
(a)<smiles>O=c1c(O)c(-c2ccc(O)c(O)c2)oc2cc(O)cc(O)c12</smiles>

(b)

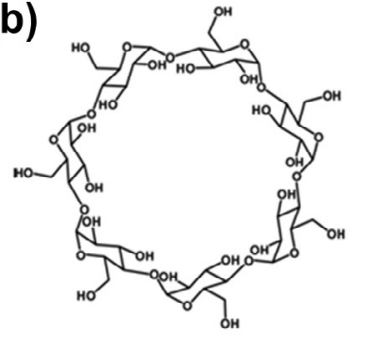

(c)

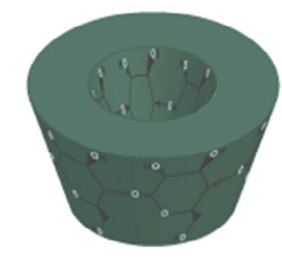

(d)

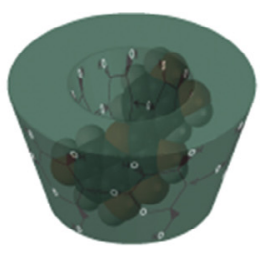

(e)

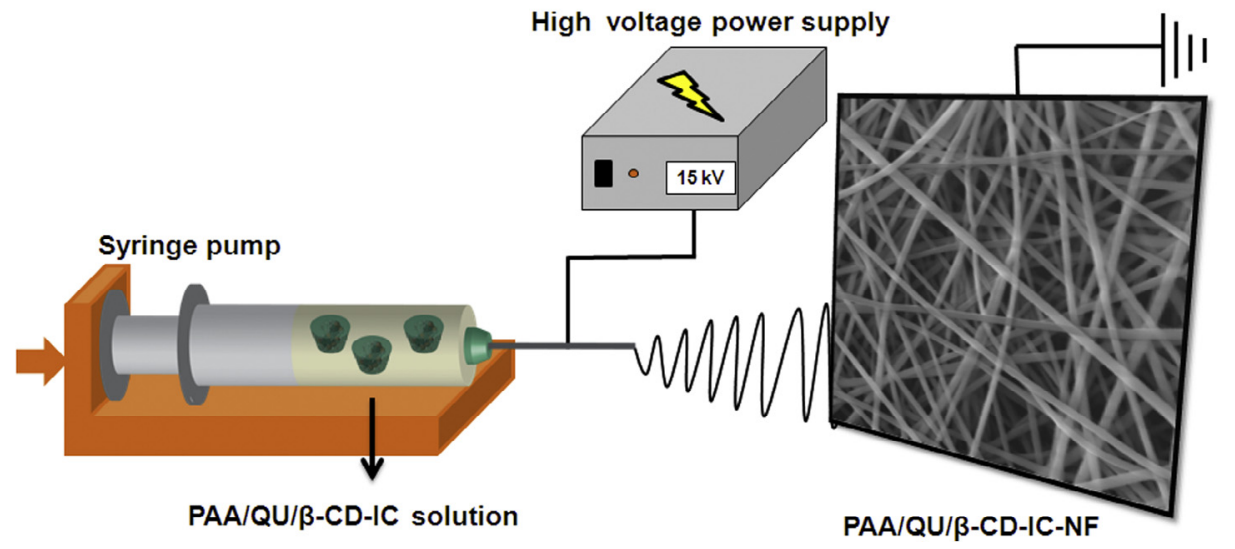

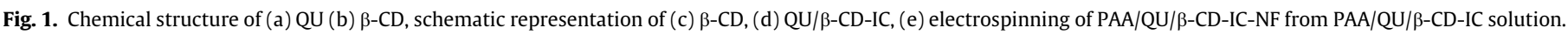

industry, pharmaceuticals, enzymology, and analytical chemistry of CDs are based on their inclusion complexation ability with a number of molecules (Del Valle, 2004). In the literature, it has been reported that QU can form IC with CDs for different purposes (Carlotti, Sapino, Ugazio, \& Caron, 2011; Zheng, Haworth, Zuo, Chow, \& Chow, 2005). In addition, incorporation of $Q U / \beta-C D-I C$ into polymeric films was also reported (Koontz, Marcy, et al., 2010; Koontz, Moffitt, et al., 2010). Incorporation of QU/ $\beta$-CD-IC into electrospun nanofibres could be a valuable approach to use QU in a more efficient way, because of the high surface area-tovolume ratio and highly porous structure of nanofibres. Related studies on CD-IC incorporated electrospun nanofibres for different purposes have already been reported by our group (Aytac, Dogan, Tekinay, \& Uyar, 2014; Aytac, Sen, Durgun, \& Uyar, 2015; Canbolat, Celebioglu, \& Uyar, 2014; Celebioglu, Umu, Tekinay, \& Uyar, 2014; Kayaci, Ertas, \& Uyar, 2013; Kayaci, Sen, Durgun, \& Uyar, 2014; Kayaci, Umu, Tekinay, \& Uyar, 2013; Kayaci \& Uyar, 2012). CDs were also used for crosslinking of polymeric materials (Li \& Hsieh, 2005; Liu, Zhang, Liu, Ni, \& Li, 2013).

In this study, an inclusion complex of $\mathrm{QU}$ and $\beta-\mathrm{CD}(\mathrm{QU} / \beta-\mathrm{CD}-$ IC) was formed at $1: 1 \mathrm{M}$ ratio (Fig. 1d) and it was incorporated into polyacrylic acid (PAA) nanofibres (PAA/QU/ $\beta-C D-I C-N F)$ via an electrospinning method (Fig. 1e). Electrospun PAA nanofibres were crosslinked by using $\beta-C D$ as a crosslinker (Li \& Hsieh, 2005). Here, $\beta-C D$ was used as a crosslinking agent for PAA nanofibres and it was also used as a host molecule for QU to form an inclusion complex. The stoichiometry of inclusion complex of QU: $\beta-C D$ was found to be a $1: 1 \mathrm{M}$ ratio by phase solubility and computational modelling studies. The inclusion complexation of QU with $\beta-C D$ considerably improved the slow release, solubility, antioxidant activity and photostability properties of QU and electrospun PAA nanofibres served as carrier matrix for $Q U / \beta-C D-I C$. $Q U / \beta-C D-I C$ incorporated PAA film (PAA/QU/ $\beta$-CD-IC-film) was also produced as a control sample. Then, PAA/QU $/ \beta-C D-I C-$ film and PAA $/ Q U / \beta$ CD-IC-NF were thermally crosslinked; thereby insoluble film and nanofibres were obtained. The structural and morphological characterisations of PAA/QU/ $\beta$-CD-IC-film and PAA/QU $/ \beta-C D-I C-N F$ were carried out using XRD and SEM, respectively. The release of $\mathrm{QU}$ from PAA/QU/ $\beta$-CD-IC-film and PAA/QU/ $\beta-C D-I C-N F$ was determined by using HPLC. The antioxidant activity of $\mathrm{QU}$, PAA/QU/ $\beta$-CD-IC-film and PAA/QU/ $\beta$-CD-IC-NF was investigated via $\mathrm{DPPH}$ radical-scavenging assay. Furthermore, in order to study the photostability, the release of $\mathrm{QU}$ from PAA/QU/ $\beta$-CD-IC-film and PAA/QU/ $\beta$-CD-IC-NF was determined by UV-Vis spectroscopy after UV light irradiation (366 nm) for $210 \mathrm{~min}$.

\section{Materials and methods}

\subsection{Materials}

Polyacrylic acid (PAA, $M_{\mathrm{w}} \sim 450.000 \mathrm{~g} / \mathrm{mol}$, Sigma-Aldrich), quercetin $(\mathrm{QU}, \geqslant 95 \%$, Sigma-Aldrich), beta-cyclodextrin $(\beta-C D$, Wacker Chemie AG, Munich, Germany), methanol (extra pure, Sigma-Aldrich), ethanol (99.8\%, Sigma-Aldrich), methanol Chromasol grade (Sigma-Aldrich), potassium phosphate monobasic (Riedel de Haen), disodium hydrogen phosphate dodecahydrate (Riedel de Haen), sodium chloride (99.0-100.5\%, Sigma Aldrich), Tween 20 (Sigma-Aldrich), 2,2-diphenyl-1-picrylhydrazyl (DPPH, Sigma-Aldrich) were purchased and used as received without any further purification. Distiled-deionised water was supplied from a Millipore Milli-Q ultrapure water system.

\subsection{Preparation of solutions}

Inclusion complex (IC) of $\mathrm{QU}$ and $\beta-\mathrm{CD}(\mathrm{QU} / \beta-\mathrm{CD}-\mathrm{IC})$ incorporating PAA film (PAA/QU/ $\beta$-CD-IC-film) and nanofibres (PAA/QU/ $\beta-C D-$ IC-NF) were produced via solvent casting and electrospinning, respectively. Initially, $5 \% \mathrm{QU}(\mathrm{w} / \mathrm{w}$, with respect to polymer) was dispersed in aqueous solution for $30 \mathrm{~min}$. Then, 19\% $\beta-C D$ (w/w, with respect to polymer) was added to give $1: 1 \mathrm{M}$ ratio (QU: $\beta-C D)$ and the solution was stirred overnight at room temperature. Finally, 7.5\% PAA (w/v) was added and the solution (PAA/QU/ $\beta-C D-I C$ solution) was stirred $4 \mathrm{~h}$ more for electrospinning. Vials were shielded from light by covering them with a piece 
of aluminium foil to prevent photochemical degradation of QU during the stirring period. For comparison, a solution of PAA $(7.5 \%, \mathrm{w} / \mathrm{v})$ was prepared in water without $\mathrm{QU}$ or $\mathrm{QU} / \beta-\mathrm{CD}-\mathrm{IC}$ (PAA solution).

\subsection{Electrospinning of nanofibres and preparation of film}

In order to produce PAA nanofibres (PAA-NF) and PAA/QU/ $\beta$-CD-IC-NF, solutions were loaded into a plastic syringe with a metallic needle (inner diameter $0.8 \mathrm{~mm}$ ) and placed horizontally on the pump (KDS101; KD Scientific, Holliston, MA). A grounded metal cylinder covered with aluminium foil was used as a collector. The distance between needle tip and collector was $15 \mathrm{~cm}$. The solution was fed at a speed of $1 \mathrm{~mL} / \mathrm{h}$ and $15 \mathrm{kV}$ was applied to the solution on the pump from the high voltage power supply (SL Series; Spellman, Hauppauge, NY) and nanofibres were collected on the aluminium foil. Electrospinning experiments were carried out in an enclosed Plexiglas box at $23-26^{\circ} \mathrm{C}$ and $18 \%$ relative humidity.

PAA/QU $/ \beta-C D-I C$ film was prepared from PAA/QU $/ \beta-C D-I C$ solution by a casting method. The same amount of $Q U, \beta-C D$ and PAA and previously described procedure were used for the preparation of the solution of PAA/QU/ $\beta$-CD-IC film. Then, the solution was put in a glass Petri dish kept in a fume hood for $48 \mathrm{~h}$; as the solvent evaporated PAA/QU/ $\beta$-CD-IC-film was obtained. Finally, PAA/QU/ $\beta$-CD-IC-film and PAA/QU/ $\beta$-CD-IC-NF were thermally crosslinked at $140{ }^{\circ} \mathrm{C}$ for $40 \mathrm{~min}$ in an oven.

\subsection{Characterisations and measurements}

\subsubsection{Phase solubility}

Phase solubility diagram was obtained according to the method reported by Higuchi and Connors (1965). An excess amount of QU ( $3 \mathrm{mg}$ ) was added to $10 \mathrm{~mL}$ of aqueous solutions of $\beta-\mathrm{CD}(0.003-$ $0.015 \mathrm{M}$ concentration range) in capped vials. The suspensions were stirred in the dark at RT for $24 \mathrm{~h}$. At equilibrium after $24 \mathrm{~h}$, withdrawn sample solutions were filtered through a $0.45-\mu \mathrm{m}$ membrane filter to remove undissolved solid and the dissolved concentration of QU was spectrophotometrically (Varian, Cary 100) determined at $375 \mathrm{~nm}$. The experiment was carried out in triplicate and the phase diagram was drawn by plotting the molar concentration of QU found in the solution against the molar concentration of $\beta-C D$. Each data point was the average of three determinations. The stability constant of the QU/CD-IC was calculated based on the phase solubility diagram according to the following equation:

$K_{\mathrm{C}}=$ slope $/ S_{\mathrm{o}}(1-$ slope $)$

where $S_{0}$ is the intrinsic solubility of QU (QU solubility in the absence of $\beta-C D$ ). The viscosity and conductivity of PAA and PAA/ $\mathrm{QU} / \beta-\mathrm{CD}-\mathrm{IC}$ solutions were measured at RT via Brookfield Viscometer DV-II+Pro equipped with cone/plate accessory using the spindle type CPE-51 and Inolab ${ }^{\circledR} \mathrm{pH} /$ Cond $720-W T W$, respectively.

\subsubsection{Computational method}

The results are obtained by first-principles calculations based on the density functional theory (Hohenberg \& Kohn, 1964; Kohn \& Sham, 1965) including Van der Waals correction (Grimme, 2006), as implemented in the Vienna Ab initio simulation package (Kresse \& Furthmüller, 1996a, 1996b). Projector augmented-wave method (PAW) (Blöchl, 1994) and a plane-wave basis set with a kinetic energy cutoff of $500 \mathrm{eV}$ were used. The exchange-correlation interaction is approximated with Perdew-Burke-Ernzerhof formalism (Perdew, Burke, \& Ernzerhof, 1996) within the generalised gradient approximation (Perdew et al., 1992). The initial structure of $\beta-C D$ was obtained from the Cambridge Structural Database (Allen, 2002). The atomic positions of the guest molecule
$(\mathrm{QU})$, bare $\beta-C D$, and their ICs were optimised using Kosugi algorithm without imposing any constraint where the total energy and atomic forces were minimised. The converge criteria for the total energy and force on each atom were set to $10^{-5} \mathrm{eV}$ and $0.01 \mathrm{eV} / \AA ̊$, respectively.

\subsubsection{Morphology analysis of nanofibres}

The morphological characterisation of PAA-NF, PAA/QU/ $\beta$-CD-IC-NF and PAA/QU/ $\beta$-CD-IC-film was performed by scanning electron microscopy (SEM; Quanta 200 FEG; FEI, Hillsboro, OR). In addition, SEM images of PAA/QU/ $\beta$-CD-IC-film and PAA/QU $/ \beta-C D-$ IC-NF were taken after crosslinking, release experiment and UV irradiation for $210 \mathrm{~min}$. Before taking SEM images, the samples were affixed to metal stubs by double-sided adhesive tape and coated with $5 \mathrm{~nm} \mathrm{Au/Pd} \mathrm{(PECS-682)} \mathrm{to} \mathrm{minimise} \mathrm{charging.} \mathrm{SEM}$ images $(n \geqslant 100)$ were analysed to calculate average fibre diameter (AFD) of nanofibres.

\subsubsection{Structural characterisation}

X-ray diffraction (XRD) data for QU (powder), PAA-NF, $\beta-C D$ (powder), PAA/QU/ $\beta$-CD-IC-film and PAA/QU/ $\beta-C D-I C-N F$ were recorded using a PANalytical X'Pert powder diffractometer applying $\mathrm{Cu} \mathrm{K} \alpha$ radiation in a $2 \theta$ range $5-30^{\circ}$, to determine the crystalline structure of the samples.

\subsubsection{In vitro release study}

Total immersion method was used to investigate the cumulative release profiles of $\mathrm{QU}$ from PAA/QU/ $\beta$-CD-IC-film and PAA/ $\mathrm{QU} / \beta$-CD-IC-NF for $48 \mathrm{~h}$. The samples $(30 \mathrm{mg}$ ) were immersed in $30 \mathrm{~mL}$ of phosphate buffered saline (PBS) including $10 \%$ methanol $(\mathrm{v} / \mathrm{v})$ and $0.5 \%$ Tween $20(\mathrm{v} / \mathrm{v})($ PBMT) at room temperature, and the solutions were stirred at $50 \mathrm{rpm}$. Aliquots $(0.5 \mathrm{~mL})$ of the test medium were withdrawn at specified time intervals and an equal amount of the fresh medium was refilled. High-performance liquid chromatography (HPLC, Agilient 1200 Series) equipped with VWD UV detector was used to measure the amount of QU released from the samples. A diol column $(50 \mathrm{~mm} \times 4.6 \mathrm{~mm}$ i.d., particle size: $5 \mu \mathrm{m}$; Inertsil GL Sciences Inc., Torrance, CA) operating at $0.75 \mathrm{~mL} / \mathrm{min}$ was used for chromatographic separation. The detection of QU was accomplished at $375 \mathrm{~nm}$ and mobile phase was $100 \%$ methanol. The calibration curve was obtained by using calibration samples prepared in PBMT. The cumulative amount of QU converted first to concentration ( $\mathrm{ppm}$ ) and then the cumulative release (\%) according to the theoretical amount of QU in PAA/QU/ $\beta$-CD-IC-film and PAA/QU/ $\beta$-CD-IC-NF. The experiments were performed in triplicate and the results were reported as average values \pm standard deviation.

\subsubsection{Antioxidant activity}

DPPH is a stable free radical that is commonly used for testing radical scavenging capability of antioxidant molecules. In its radical scavenging mechanism, the antioxidant molecule acts as a hydrogen donor that transforms DPPH radical into its reduced form. Therefore radical character of DPPH is neutralised (DPPHH) and its colour turns from purple to yellow (Mishra, Ojha, \& Chaudhury, 2012). Antioxidant activity of QU (powder) and after its incorporation into PAA/QU/ $\beta$-CD-IC-film and PAA/QU $/ \beta-C D-I C$ NF was determined via 2,2-diphenyl-1-picrylhydrazyl (DPPH) radical scavenging assay. Five milligrams of PAA/QU $/ \beta-C D-I C-f i l m$, PAA/QU/ $\beta$-CD-IC-NF and theoretically corresponding amount of QU were immersed in $3 \mathrm{~mL}$ of $10^{-4} \mathrm{M}$ DPPH solution prepared in ethanol/water (50:50) solution. The resulting mixtures were incubated in the dark at room temperature. After the system reached equilibrium at the end of $60 \mathrm{~min}$, the absorbances of the solutions were measured by UV-Vis spectroscopy (Varian Cary 100) at $525 \mathrm{~nm}$. 
\%of DPPH scavenging $=100 \times\left(A_{\mathrm{B}}-A_{\mathrm{S}}\right) / A_{\mathrm{B}}$

where $A_{\mathrm{B}}$ is the absorption of the blank and $A_{\mathrm{S}}$ is the absorption of the sample.

\subsubsection{Photostability}

In order to investigate the photostability of $\mathrm{QU}$ in PAA/QU/ $\beta$ CD-IC-film and PAA/QU/ $\beta-C D-I C-N F$, the samples were mounted $10 \mathrm{~cm}$ away from a UV lamp ( $8 \mathrm{~W}$, Camag) and irradiated with $366 \mathrm{~nm}$ UV light during pre-determined time intervals over 210 min. After irradiation, PAA/QU/ $\beta$-CD-IC-film and PAA/QU/ $\beta$ CD-IC-NF were immersed in PBMT and the amount of QU released was measured at the end of $48 \mathrm{~h}$ by UV-Vis spectroscopy at $375 \mathrm{~nm}$. Each sample was analysed in triplicate and the results are given comparative to QU released from non-irradiated PAA/ $\mathrm{QU} / \beta-\mathrm{CD}-\mathrm{IC}$-film and PAA/QU/ $\beta$-CD-IC-NF as average \pm standard values.

\section{Results and discussion}

\subsection{Phase solubility study}

The phase solubility analysis is a widely used method for determining the effect of $C D$ complexation on the solubility of guest molecules. The diagram is obtained by measuring the molar concentration of the guest in the presence of $C D$ at various concentrations. The phase solubility diagram obtained for $Q U / \beta-C D$ system is shown in Fig. S1 in the Supplementary material. The solubility of QU linearly increased as a function of $\beta-C D$ concentration. The enhancement in the solubility of QU confirms a certain degree of $\mathrm{CD}$-IC formation. Furthermore, the profile of the diagram corresponds to $A_{\mathrm{L}}$ type, indicating 1:1 molecular complex formation. The stability constant $\left(K_{\mathrm{c}}\right)$ of host-guest complex is an indication of the binding strength between $\mathrm{CD}$ and guest molecule. $K_{\mathrm{c}}$ calculated for $\mathrm{QU} / \beta$-CD-IC was $3345 \mathrm{M}^{-1}$, suggesting a relatively moderate interaction (Takahashi, Veiga, \& Ferraz, 2012). To the best of our knowledge, the observed $K c$ of $Q U / \beta-C D-I C$ is higher than in an earlier report (Zheng et al., 2005).

\subsection{Molecular modelling of $Q U / \beta-C D-I C$}

To clarify the inclusion process of QU within the $\beta$-CD cavity, the initial structures of $\beta-\mathrm{CD}$ and $\mathrm{QU}$ molecules are optimised under vacuum and thus ground state geometries are obtained. Subsequently, a single QU molecule is introduced into the cavity of $\beta-C D$ at various positions with different orientations. The three possible orientations of $\mathrm{QU}$ are labelled by its rings (A, B, C) (Fiorucci, Golebiowski, Cabrol-Bass, \& Antonczak, 2007) and are shown in Fig. 2a. Anticipated by the size matching, A and B orientations can form a complex (Fig. $2 \mathrm{~b}$ and $\mathrm{c}$ ) while $\mathrm{C}$ orientation cannot fit into $\beta$-CD. The complexation energy $\left(E_{\text {comp }}\right)$ for $1: 1$ (QU: $\beta-\mathrm{CD})$ stoichiometry can be calculated as:

$E_{\text {comp }}=E_{\mathrm{CD}}+E_{\text {guest }}-E_{\mathrm{IC}}$

where $E_{\mathrm{CD}}, E_{\text {guest }}$, and $E_{\mathrm{IC}}$ is the total energy of $\beta-\mathrm{CD}, \mathrm{QU}$, and QU/ $\beta$-CD-IC, respectively. To compare $E_{\text {comp }}$ depending on the relative position of the rings in $\beta-C D, Q U$ was placed in different positions with respect to the centre of mass of QU. For the lowest energy configurations (Fig. 2b and c), $E_{\text {comp }}$ is calculated to be 22.8 and $20.5 \mathrm{kcal} / \mathrm{mol}$ for A and B orientation, respectively. Although A orientation is energetically preferred, our analysis reveals that the change in $E_{\text {comp }}$ is not drastic, suggesting no significant preference in the orientation. These results indicate that QU has the possibility to penetrate the cavity of $\beta$-CD through either ring $A$ or ring $B$. Further, 2:1 stoichiometry is also considered, but two QU molecules do not fit into one $\beta-C D$ and thus cannot form IC. On the other hand, considering the fact that A and B orientation yields similar $E_{\text {comp, }}$ single QU is long enough to show a tendency to form IC with two $\beta$-CD molecules. To test this presumption, a case where single QU molecule is captured by two $\beta-C D$ molecules from $A$ and $B$ rings was also modelled and shown in Fig. 2 d. $E_{\text {comp }}$ for this $(1: 2)$ stoichiometry is calculated to be $30.6 \mathrm{kcal} / \mathrm{mol}$ and it is even higher than (1:1) stoichiometry. (For this case the total energy of two $\beta-C D$ molecules is considered and CD-CD interaction is also taken into account). Such complexation can be formed when the second $\beta$-CD approaches CD-IC along the A or B ring while the other ring is already inside the cavity of the former $\beta-C D$. As expected the energy is not altered significantly for both cases indicating also no orientation preference. The binding energy of second $\beta-C D$ from $A$ and $\mathrm{B}$ is calculated to be 12.1 and $13.0 \mathrm{kcal} / \mathrm{mol}$, respectively.

To further elucidate the affinity towards the guest molecule, QU molecule is initially placed $4 \AA$ away from the centre of the $\beta-C D$ cavity and then moved towards $\beta-C D$ in $2.0 \AA$ steps until the $C$ rings of QU are fully placed in the $\beta-C D$ cavity. At each moving step, the IC is optimised apart from considering the coordinates of the incoming QU molecule in the moving direction. The corresponding energy path does not indicate any energy barrier and suggests that IC formation is exothermic. As $E_{\text {comp }}$ is high both for (1:1) and (1:2) stoichiometry, IC is expected to be stable at ambient conditions. However, 1:1 stoichiometry was preferred in order to have higher weight loading of QU in the PAA nanofibres.

\subsection{Morphology analysis of nanofibres}

Representative SEM images of PAA-NF and PAA/QU/ $\beta$-CD-IC-NF are shown in Fig. 3. PAA-NF and PAA/QU/ $\beta$-CD-IC-NF were beadfree and uniform and have average diameters of $435 \pm 90 \mathrm{~nm}$ and $270 \pm 50 \mathrm{~nm}$, respectively. The changes in viscosity and conductivity of the solutions affect the diameter of the electrospun nanofibres (Bhardwaj \& Kundu, 2010). The conductivities of PAA $(1678 \mu \mathrm{S} / \mathrm{cm})$ and PAA/QU/ $\beta$-CD-IC $(1583 \mu \mathrm{S} / \mathrm{cm})$ solutions were almost same; however the viscosity of PAA/QU/ $\beta$-CD-IC solution $(0.113 \mathrm{~Pa} \cdot \mathrm{s})$ was lower than PAA solution $(0.129 \mathrm{~Pa} \cdot \mathrm{s})$, which is why the diameter of PAA/QU/ $\beta$-CD-IC-NF was smaller compared to PAA-NF. The SEM image of PAA/QU/ $\beta$-CD-IC-film showed its smooth structure without pores and cracks (Fig. S2, Supplementary material). In addition, the crystals observed in the SEM image might be due to the presence of free $Q U$ molecules in PAA/QU/ $\beta$ CD-IC-film. Moreover, SEM images of PAA/QU $/ \beta-C D-I C-f i l m$ and PAA/QU/ $\beta$-CD-IC-NF after crosslinking at $140^{\circ} \mathrm{C}$ for $40 \mathrm{~min}$ are shown in Fig. S3 in the Supplementary material. As seen from the SEM images, PAA/QU/ $\beta$-CD-IC-film and PAA/QU $/ \beta-C D-I C-N F$ did not show any appreciable change in the surface morphology due to the crosslinking. Stability tests of thermally crosslinked $\mathrm{PAA} / \mathrm{QU} / \beta-\mathrm{CD}-\mathrm{IC}-$ film and PAA/QU/ $\beta$-CD-IC-NF were carried out by soaking the nanofibres in water for $48 \mathrm{~h}$. PAA/QU/ $\beta$-CD-IC-film and PAA/QU/ $\beta$-CD-IC-NF did not dissolve in water. These results showed that sufficient crosslinking had occurred in the film and nanofibre samples.

\subsection{Structural characterisation}

The XRD patterns of QU powder, PAA-NF, $\beta-C D$ powder, PAA/ $\mathrm{QU} / \beta$-CD-IC-film and PAA/QU/ $\beta$-CD-IC-NF web are displayed in Fig. 4. As seen from XRD diffractograms, QU is a crystalline compound with characteristic diffraction peaks. The amorphous nature of PAA was observed in the XRD pattern of PAA-NF. The native CDs have cage-type packing crystal structure and the cavity of each $C D$ molecule is blocked by other CD molecules. Received native CDs have characteristic peaks in the range of $2 \theta=5-30^{\circ}$. When an inclusion complex is formed, guest molecules cannot form crystals since they are separated from each other by the cavity of the CD 
(a)

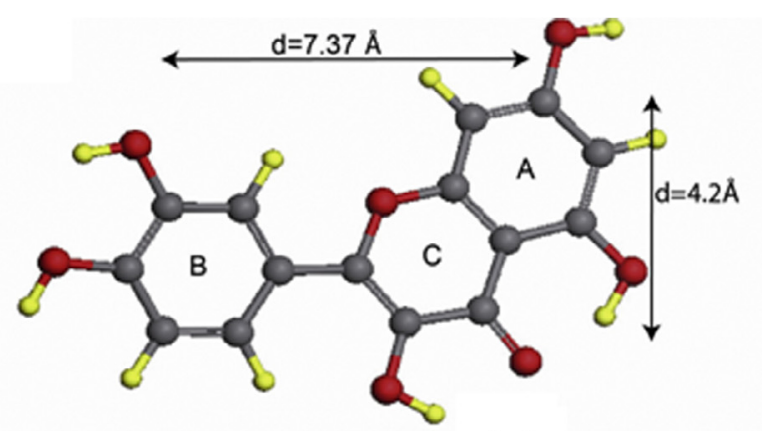

(b)

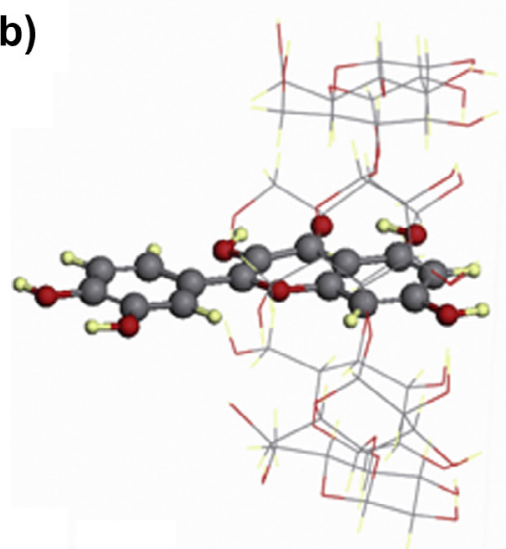

(c)

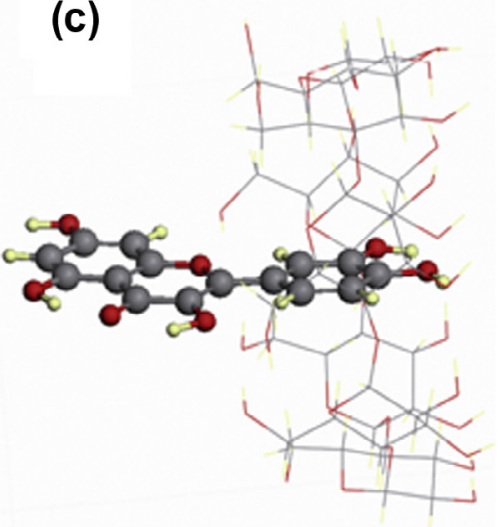

(d)

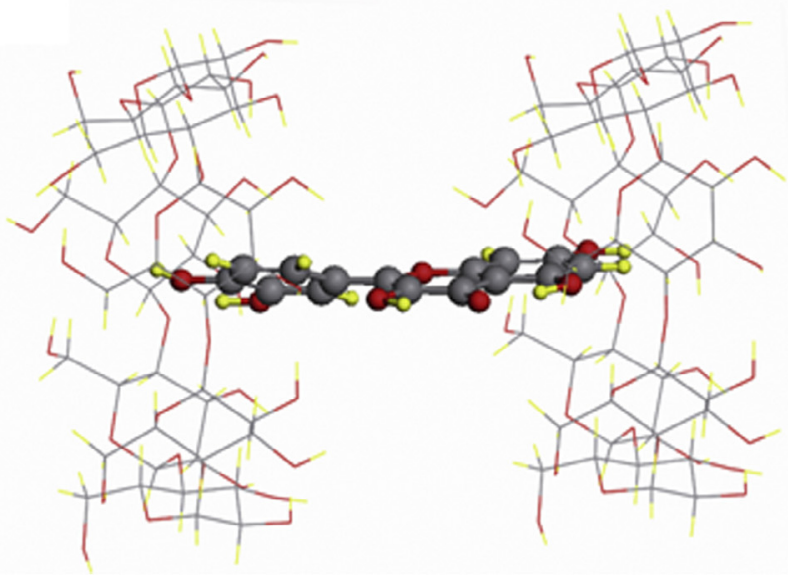

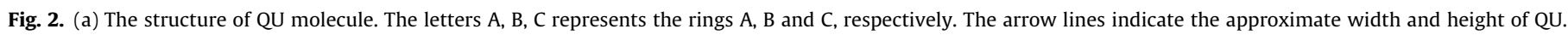

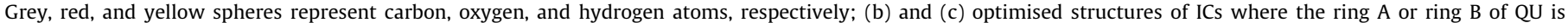

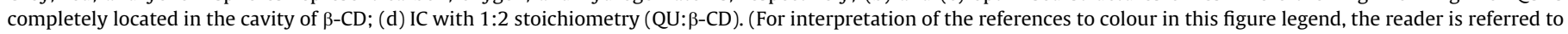
the web version of this article.)

molecules (Celebioglu \& Uyar, 2011). PAA/QU/ $\beta-C D-I C-N F$ exhibited an amorphous XRD pattern without showing the crystalline peaks of $\mathrm{QU}$, due to the complexation between $\mathrm{QU}$ and $\beta-C D$. However, PAA/QU/ $\beta$-CD-IC-film exhibited most of the crystalline peaks of $\mathrm{QU}$. Therefore, there are some free $\mathrm{QU}$ molecules in PAA/QU/ $\beta$-CD-IC-film.

\subsection{In vitro release study}

The release of QU from PAA/QU/ $\beta-C D-I C-N F$ into PBS buffer was tested at room temperature using HPLC. In this case, the release of QU into PBS could not be measured. Methanol and Tween 20 were added into PBS to give PBMT which could facilitate detection of QU. The cumulative release (\%) of QU into PBMT versus time is shown in Fig. 5. So far, a number of investigations strongly reported the limited release capability of QU into various media. QU has not been released from polymeric films into coconut oil or $95 \%$ ethanol because of its limited solubility (Koontz, Moffitt, et al., 2010). It was also concluded that the low compatibility between QU and the release medium (water) reduced the extent of release considerably (López-de-Dicastillo, Alonso, Catalá, Gavara, \& HernándezMuñoz, 2010; López-de-Dicastillo, Gómez-Estaca, Catalá, Gavara, \& Hernández-Muñoz, 2012). On the other hand, the release of QU has been observed into water and physiological saline (Dias et al., 2011; Li et al., 2014). According to another study the release of QU (into 95\% ethanol) depends on the hydrophobicity of polymers. In hydrophobic polymers, hydroxyl groups in their structure prevent miscibility of QU in the polymer and a tendency of QU molecules to cluster together. The presence of hydrophilic polymer enables QU molecule to have comparatively high mobility. Since hydroxyl groups make QU more miscible whereas the hydrophobic ring prevents binding of QU molecules to the polymer (Chen, Lee, Zhu, \& Yam, 2012). The release of QU from both PAA/QU/ $/ \mathrm{CD}$ IC-film and PAA/QU- $\beta$-CD-IC-NF was achieved due to the solubility 

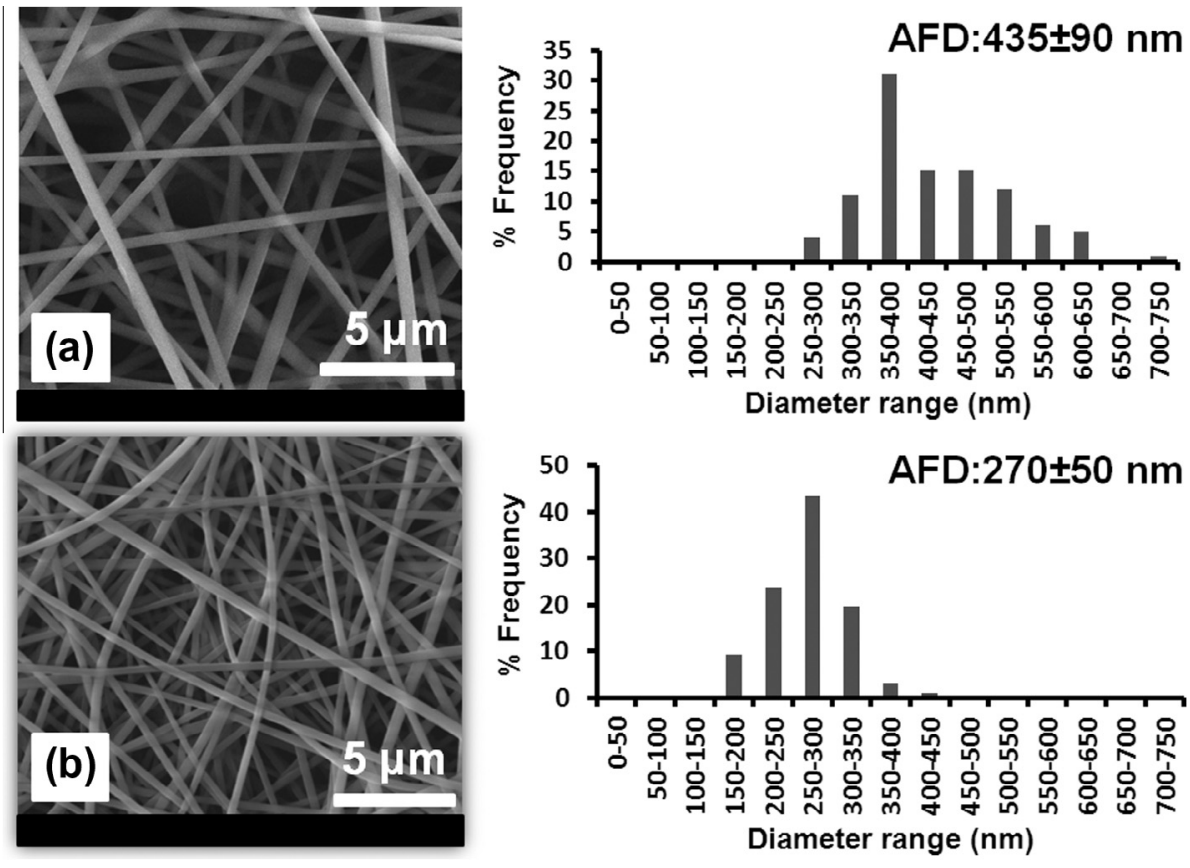

Fig. 3. SEM images of (a) PAA-NF and (b) PAA/QU/ $\beta-C D-I C-N F$ (before crosslinking).

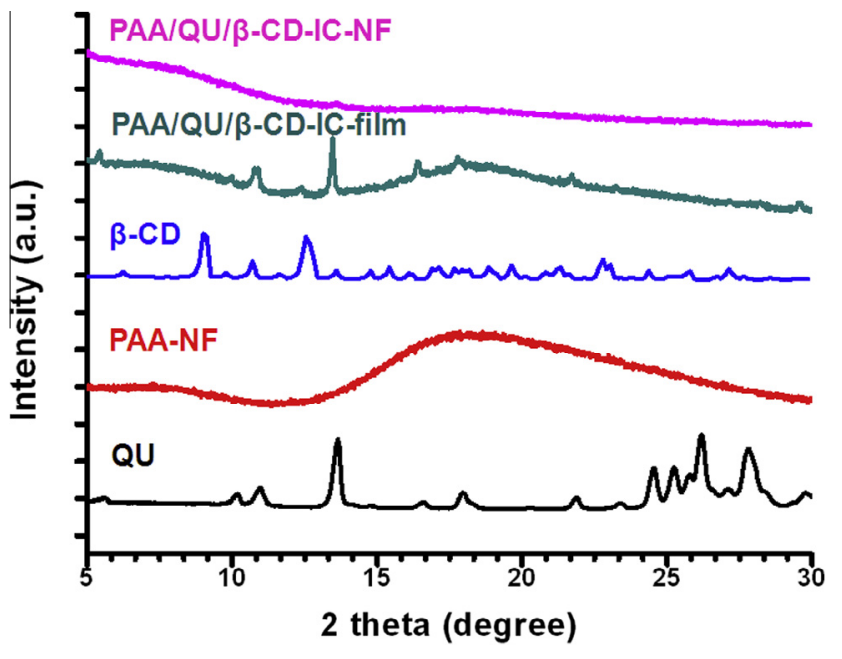

Fig. 4. XRD patterns of $Q U, P A A-N F, \beta-C D, P A A / Q U / \beta-C D-I C-f i l m$ and $P A A / Q U / \beta-C D-$ IC-NF.

increment of QU by $\mathrm{CD}-\mathrm{IC}$ shown in phase solubility results. The release kinetic of QU into PBMT from PAA/QU/ $\beta$-CD-IC-film and PAA/QU/ $\beta$-CD-IC-NF can be divided into two stages: an initial fast release and then a relatively slow release. The release of $\mathrm{QU}$ from $\mathrm{PAA} / \mathrm{QU} / \beta-\mathrm{CD}-\mathrm{IC}-\mathrm{NF}$ was slower than PAA/QU/ $\beta$-CD-IC-film. Thus, PAA/QU/ $\beta$-CD-IC-NF released $57 \%$ of the theoretically loaded QU into PBMT within $30 \mathrm{~min}$ of immersion, whereas PAA/QU/ $\beta$-CD-IC-film released $77 \%$ of the theoretically loaded QU over the same time. This might be related with the presence of free QU molecules, as shown in XRD results and QU/ $\beta$-CD-IC molecules close to the surface, due to the slow evaporation of solvent during formation of PAA/QU $/ \beta-C D-I C-$ film. However, the total released amount of QU into PBMT in $48 \mathrm{~h}$ was much more from PAA/QU- $\beta$-CD-IC-NF (97\%, with respect to the theoretically loaded $\mathrm{QU})$ than PAA/QU/ $\beta$-CD-IC-film (79\%, with respect to the theoretically loaded $\mathrm{QU}$ ). The possible reasons for this might be the high surface area of PAA/QU- $\beta$-CD-IC-NF increasing the contact of QU

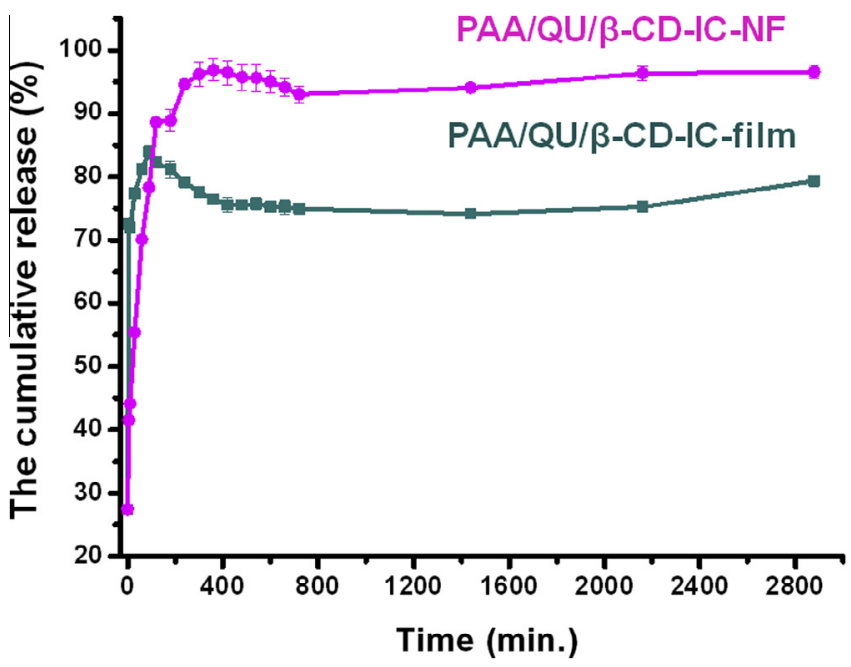

Fig. 5. The cumulative release of $Q U$ from $P A A / Q U / \beta-C D-I C-$ film and $P A A / Q U / \beta-C D-$ IC-NF $(n=3)$. The error bars in the figure represent the standard deviation (SD).

with the release medium and the existence of free QU molecules in PAA/QU/ $\beta$-CD-IC-film. As a conclusion, controlled release of $\mathrm{QU}$ has been successfully achieved from PAA/QU- $\beta$-CD-IC-NF into PBMT. Furthermore, the morphology of PAA/QU/ $\beta$-CD-IC-film and $\mathrm{PAA} / \mathrm{QU} / \beta-\mathrm{CD}-\mathrm{IC}-\mathrm{NF}$ were preserved after the release experiments performed in PBMT (Fig. S3, Supplementary material). This is most likely due to the thermal crosslinking. For PAA/QU $/ \beta-C D-I C-$ film and PAA/QU/ $\beta$-CD-IC-NF, hydroxyl groups of $\beta-C D$ that are outside the cavity and carboxyl groups of PAA were crosslinked and insoluble PAA/QU/ $\beta$-CD-IC-film and PAA/QU/ $\beta$-CD-IC-NF were obtained.

\subsection{Antioxidant activity}

Antioxidant property of QU originates from three functional groups in the structure: $3^{\prime}, 4^{\prime}$-dihydroxycatechol configuration in the B-ring, 2,3-double bond with the 4-carbonyl group and 
3-hydroxy group with the 2,3-double bond, both in the C-ring (Smith, Thomsen, Markham, Andary, \& Cardon, 2000). DPPH is usually dissolved in alcohols like methanol and ethanol; however, the hydrophobic medium of alcohols reduces the hydrophobic driving force for complexation. Therefore, the amount of ethanol was reduced up to $50 \%$ while dissolving DPPH. Further reducing the amount of ethanol results in coagulation of DPPH. The photographs of the DPPH solution before reaction; QU, PAA/QU/ $\beta$-CD-IC-film and PAA/QU/ $\beta-C D-I C-N F$ after the reaction are shown in Fig. $6 a$. According to the results of the modelling study, QU was partially included in the cavity of $\beta-C D$, so it could maintain its antioxidant activity after complexation (Carlotti et al., 2011). As seen from the photographs, the colour of QU, PAA/QU/ $\beta-C D-I C-$ film and PAA/QU/ $\beta$-CD-IC-NF including solutions were all yellow. The antioxidant activity (\%) of QU, PAA/QU/ $\beta$-CD-IC-film and PAA/QU/ $\beta$-CD-IC-NF were calculated as $85 \pm 1.51 \%, 95 \pm 0.69 \%$ and $94 \pm 0.61 \%$, respectively. QU exhibited lower antioxidant activity compared to $P A A / Q U / \beta-C D-I C-$ film and PAA/QU/ $\beta-C D-I C-N F$. This is most likely due to the limited solubility of QU (powder) in ethanol/water (50:50) solution and enhanced solubility of QU in aqueous solution by complexation. The amount of QU used for the preparation of $\mathrm{QU} / \beta-\mathrm{CD}-\mathrm{IC}$ was almost identical for the film and nanofibre samples, so the antioxidant activities of PAA/QU/ $\beta-C D-I C-$ film and $\mathrm{PAA} / \mathrm{QU} / \beta-\mathrm{CD}-\mathrm{IC}-\mathrm{NF}$ were very close each other. But as mentioned above, the total released amount of QU into PBMT was much more from PAA/QU- $\beta$-CD-IC-NF compared to PAA/QU/ $\beta$-CD-IC-film. The reason of comparable antioxidant activity of $P A A / Q U / \beta-C D-I C-$ film and PAA/QU/ $\beta$-CD-IC-NF might be the higher solubility of $\mathrm{QU}$ in ethanol/water (50:50) media compared to PBMT. In addition, QU maintained its antioxidant activity in PAA/QU/ $\beta$-CD-IC-NF even after applying high electrical potential during the electrospinning process.

\subsection{Photostability}

In general, QU might undergo photooxidation upon UV irradiation (Carlotti et al., 2011; Smith et al., 2000). However, photostability is of vital importance for QU to show its activity. CD is known to provide protection for guest molecules against visible or UV light (Del Valle, 2004). In order to investigate the protective effect of CD-IC on the photostability of QU, UV irradiation tests were performed for PAA/QU $/ \beta-C D-I C-$ film and PAA/QU $/ \beta-C D-I C-N F$ (Fig. 6b). The release of $\mathrm{QU}$ from $\mathrm{UV}$-irradiated PAA/QU/ $\beta$-CD-IC-film and
PAA/QU/ $\beta$-CD-IC-NF for $210 \mathrm{~min}$ was determined to be $75.46 \pm 0.13 \%$ and $74.40 \pm 0.06 \%$ comparative to non-irradiated $\mathrm{PAA} / \mathrm{QU} / \beta-\mathrm{CD}-\mathrm{IC}$-film and PAA/QU/ $\beta$-CD-IC-NF, respectively. CDICs provide a more apolar environment that reduces the photolytic reaction and the existence of a screening effect also causes some photostability, owing to the prevention of the passage of light to the guest molecule (Carlotti et al., 2011). The hydroxyl group on the $\mathrm{C}$ ring is known to be the main determinant for the photostability of QU (Smith et al., 2000). According to the modelling study, this group was not completely in the cavity of $\beta-C D$, so the protection of $\mathrm{CD}$-IC could be moderate. However, the reason for the protection might not only be the presence of CD-IC in PAA/QU $/ \beta-C D-I C$-film and $\mathrm{PAA} / \mathrm{QU} / \beta-\mathrm{CD}-\mathrm{IC}-\mathrm{NF}$, the crosslinked nanofibrous structure could also have an effect on the protection of QU against UV irradiation. It has been suggested that intermolecular hydrogen bonds reinforced the structure, and energy of radiation is partially used for breaking the hydrogen bonds (Kaczmarek, Szalla, \& Kamińska, 2001). Moreover, UV irradiation did not result in any change in the morphology of PAA/QU/ $\beta-C D-I C-$ film and PAA/QU $/ \beta-C D-I C-N F$ (Fig. S3, Supplementary material).

\section{Conclusion}

In conclusion, QU was included in the $\beta-C D$ cavity and then incorporated into PAA solution and PAA/QU/ $\beta$-CD-IC-NF was successfully produced via electrospinning. Then, PAA/QU/ $\beta-C D-I C-N F$ were crosslinked thermally and became a water-insoluble web. Here, $\beta$-CD was used as not only the host molecule for QU but also as a crosslinking agent for the PAA-NF matrix. Phase solubility and modelling studies suggested the stoichiometry of $Q U: \beta-C D$ was $1: 1$. Phase solubility studies also revealed that solubility of QU was increased by inclusion complexation. The electrospinning of PAA/QU/ $\beta$-CD-IC resulted in bead-free and uniform nanofibres having diameter of $270 \pm 50 \mathrm{~nm}$, as confirmed by SEM imaging. The crystalline peaks of QU were not detected in the XRD pattern of PAA/QU/ $\beta$-CD-IC-NF, possibly due to complexation between $Q U$ and $\beta-C D$. The release of QU from PAA/QU $/ \beta-C D-I C-N F$ was slower and higher in total compared to PAA/QU/ $\beta-C D-I C$-film. In addition, PAA/QU/ $\beta$-CD-IC-NF exhibited quite high antioxidant activity and photostability. As a result, a PAA/QU/ $\beta-C D-I C-N F$ web showing a slow release profile of QU with rather high antioxidant activity and photostability can be achievable, due to its high surface area and high porosity.

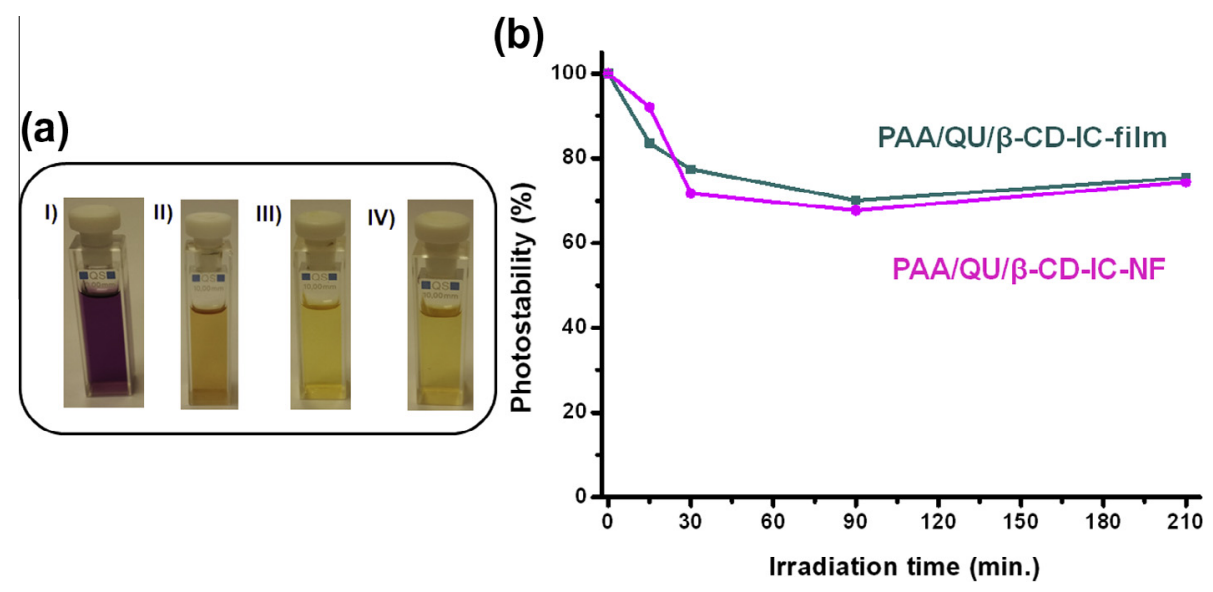

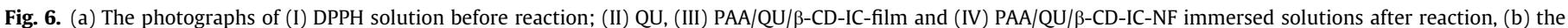

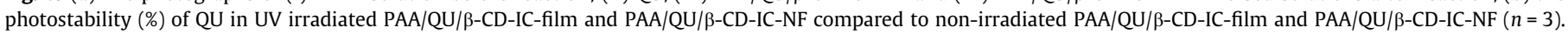
The error bars in the figure represent the standard deviation (SD). 


\section{Acknowledgements}

This work is supported by The Scientific and Technological Research Council of Turkey (TUBITAK) (Project \# 111M459). Dr. Uyar and Dr. Durgun acknowledge partial support from The Turkish Academy of Sciences - Outstanding Young Scientists Award Program (TUBA-GEBIP). Z. Aytac thanks to TUBITAK (project \#111M459 and \#213M185) for the PhD scholarship.

\section{Appendix A. Supplementary data}

Supplementary data associated with this article can be found, in the online version, at http://dx.doi.org/10.1016/j.foodchem.2015. 11.051 .

\section{References}

Agarwal, S., Wendorff, J. H., \& Greiner, A. (2008). Use of electrospinning technique for biomedical applications. Polymer, 49, 5603-5621.

Allen, F. H. (2002). The Cambridge structural database: A quarter of a million crystal structures and rising. Acta Crystallographica Section B: Structural Science, 58(3), 380-388.

Aytac, Z., Dogan, S. Y., Tekinay, T., \& Uyar, T. (2014). Release and antibacterial activity of allyl isothiocyanate/beta-cyclodextrin complex encapsulated in electrospun nanofibers. Colloids and Surfaces B: Biointerfaces, 120, 125-131.

Aytac, Z., Sen, H. S., Durgun, E., \& Uyar, T. (2015). Sulfisoxazole/cyclodextrin inclusion complex incorporated in electrospun hydroxypropyl cellulose nanofibers as drug delivery system. Colloids and Surfaces B: Biointerfaces, 128, 331-338.

Bhardwaj, N., \& Kundu, S. C. (2010). Electrospinning: A fascinating fiber fabrication technique. Biotechnology Advances, 28, 325-347.

Blöchl, P. E. (1994). Projector augmented-wave method. Physical Review B, 50(24), 17953.

Canbolat, M. F., Celebioglu, A., \& Uyar, T. (2014). Drug delivery system based on cyclodextrin-naproxen inclusion complex incorporated in electrospun polycaprolactone nanofibers. Colloid Surface B: Biointerfaces, 115, 15-21.

Carlotti, M. E., Sapino, S., Ugazio, E., \& Caron, G. (2011). On the complexation of quercetin with methyl- $\beta$-cyclodextrin: Photostability and antioxidant studies. Journal of Inclusion Phenomena and Macrocyclic, 70(1-2), 81-90.

Celebioglu, A., Umu, O. C., Tekinay, T., \& Uyar, T. (2014). Antibacterial electrospun nanofibers from triclosan/cyclodextrin inclusion complexes. Colloids and Surfaces B: Biointerfaces, 116, 612-619.

Celebioglu, A., \& Uyar, T. (2011). Electrospinning of polymer-free nanofibers from cyclodextrin inclusion complexes. Langmuir, 27, 6218-6226.

Chen, X., Lee, D. S., Zhu, X., \& Yam, K. L. (2012). Release kinetics of tocopherol and quercetin from binary antioxidant controlled-release packaging films. Journal of Agricultural and Food Chemistry, 60(13), 3492-3497.

Del Valle, E. M. M. (2004). Cyclodextrins and their uses: A review. Process Biochemistry, 39(9), 1033-1046.

Dias, A., Braga, M., Seabra, I., Ferreira, P., Gil, M., \& De Sousa, H. (2011). Development of natural-based wound dressings impregnated with bioactive compounds and using supercritical carbon dioxide. International Journal of Pharmaceutics, 408(1) 9-19.

Fiorucci, S., Golebiowski, J., Cabrol-Bass, D., \& Antonczak, S. (2007). DFT study of quercetin activated forms involved in antiradical, antioxidant, and prooxidant biological processes. Journal of Agricultural and Food Chemistry, 55(3), 903-911.

Grimme, S. (2006). Semiempirical GGA-type density functional constructed with a long-range dispersion correction. Journal of Computational Chemistry, 27(15), 1787-1799.

Higuchi, T., \& Connors, K. A. (1965). Phase-solubility techniques. Advances in Analytical Chemistry and Instrumentation, 4(2), 117-212.

Hohenberg, P., \& Kohn, W. (1964). Inhomogeneous electron gas. Physical Review, 136 (3B), B864.

Kaczmarek, H., Szalla, A., \& Kamińska, A. (2001). Study of poly (acrylic acid)-poly (vinylpyrrolidone) complexes and their photostability. Polymer, 42(14), 6057-6069.
Kayaci, F., Ertas, Y., \& Uyar, T. (2013). Enhanced thermal stability of eugenol by cyclodextrin inclusion complex encapsulated in electrospun polymeric nanofibers. Journal of Agricultural and Food Chemistry, 61(34), 8156-8165.

Kayaci, F., Sen, H. S., Durgun, E., \& Uyar, T. (2014). Functional electrospun polymeric nanofibers incorporating geraniol-cyclodextrin inclusion complexes: High thermal stability and enhanced durability of geraniol. Food Research International, 62, 424-431.

Kayaci, F., Umu, O. C. O., Tekinay, T., \& Uyar, T. (2013). Antibacterial electrospun poly(lactic acid) (PLA) nanofibrous webs incorporating triclosan/cyclodextrin inclusion complexes. Journal of Agricultural and Food Chemistry, 61(16), 3901-3908.

Kayaci, F, \& Uyar, T. (2012). Encapsulation of vanillin/cyclodextrin inclusion complex in electrospun polyvinyl alcohol (PVA) nanowebs: Prolonged shelf-life and high temperature stability of vanillin. Food Chemistry, 133(3), 641-649.

Kohn, W., \& Sham, L. J. (1965). Self-consistent equations including exchange and correlation effects. Physical Review, 140(4A), A1133.

Koontz, J. L., Marcy, J. E., O’Keefe, S. F., Duncan, S. E., Long, T. E., \& Moffitt, R. D. (2010). Polymer processing and characterization of LLDPE films loaded with alpha-tocopherol, quercetin, and their cyclodextrin inclusion complexes. Journal of Applied Polymer Science, 117(4), 2299-2309.

Koontz, J. L., Moffitt, R. D., Marcy, J. E., O'Keefe, S. F., Duncan, S. E., \& Long, T. E, (2010). Controlled release of $\alpha$-tocopherol, quercetin, and their cyclodextrin inclusion complexes from linear low-density polyethylene (LLDPE) films into a coconut oil model food system. Food Additives and Contaminants - Part A Chemistry, Analysis, Control, Exposure and Risk Assessment, 27(11), 1598-1607.

Kresse, G., \& Furthmüller, J. (1996a). Efficient iterative schemes for ab initio totalenergy calculations using a plane-wave basis set. Physical Review B, 54(16), 11169.

Kresse, G., \& Furthmüller, J. (1996b). Efficiency of ab-initio total energy calculations for metals and semiconductors using a plane-wave basis set. Computational Materials Science, 6(1), 15-50.

Li, L., \& Hsieh, Y.-L. (2005). Ultra-fine polyelectrolyte fibers from electrospinning of poly (acrylic acid). Polymer, 46(14), 5133-5139.

Li, C., Wang, Z.-H., \& Yu, D.-G. (2014). Higher quality quercetin sustained release ethyl cellulose nanofibers fabricated using a spinneret with a Teflon nozzle. Colloids and Surfaces B: Biointerfaces, 114, 404-409.

Liu, C., Zhang, Z., Liu, X., Ni, X., \& Li, J. (2013). Gelatin-based hydrogels with $\beta$ cyclodextrin as a dual functional component for enhanced drug loading and controlled release. RSC Advances, 3(47), 25041-25049.

López-de-Dicastillo, C., Alonso, J. M., Catalá, R., Gavara, R., \& Hernández-Muñoz, P. (2010). Improving the antioxidant protection of packaged food by incorporating natural flavonoids into ethylene-vinyl alcohol copolymer (EVOH) films. Journal of Agricultural and Food Chemistry, 58(20), 10958-10964.

López-de-Dicastillo, C., Gómez-Estaca, J., Catalá, R., Gavara, R., \& Hernández-Muñoz, P. (2012). Active antioxidant packaging films: Development and effect on lipid stability of brined sardines. Food Chemistry, 131(4), 1376-1384.

Mercader-Ros, M. T., Lucas-Abellán, C., Fortea, M. I., Martínez-Cachá, A., Gabaldón, J. A., \& Núñez-Delicado, E. (2012). Quercetin: Dietary sources, functions and health benefits. Nova Science Pub Inc., pp. 179-198.

Mishra, K., Ojha, H., \& Chaudhury, N. K. (2012). Estimation of antiradical properties of antioxidants using DPPH assay: A critical review and results. Food Chemistry, 130(4), 1036-1043.

Perdew, J. P., Burke, K., \& Ernzerhof, M. (1996). Generalized gradient approximation made simple. Physical Review Letters, 77(18), 3865.

Perdew, J. P., Chevary, J., Vosko, S., Jackson, K. A., Pederson, M. R., Singh, D., \& Fiolhais, C. (1992). Atoms, molecules, solids, and surfaces: Applications of the generalized gradient approximation for exchange and correlation. Physical Review B, 46(11), 6671.

Smith, G. J., Thomsen, S. J., Markham, K. R., Andary, C., \& Cardon, D. (2000). The photostabilities of naturally occurring 5-hydroxyflavones, flavonols, their glycosides and their aluminium complexes. Journal of Photochemistry and Photobiology A: Chemistry, 136(1), 87-91.

Takahashi, A. I., Veiga, F. J. B., \& Ferraz, H. G. (2012). A literature review of cyclodextrin inclusion complexes characterization - Part I: Phase solubility diagram, dissolution and scanning electron microscopy. International Journal of Pharmaceutical Sciences Review and Research, 12(1), 1-6.

Xing, Z.-C., Meng, W., Yuan, J., Moon, S., Jeong, Y., \& Kang, I.-K. (2012). In vitro assessment of antibacterial activity and cytocompatibility of quercetincontaining PLGA nanofibrous scaffolds for tissue engineering. Journal of Nanomaterials, 1, 1-7.

Zheng, Y., Haworth, I. S., Zuo, Z., Chow, M. S., \& Chow, A. H. (2005). Physicochemical and structural characterization of quercetin- $\beta$-cyclodextrin complexes. Journal of Pharmaceutical Sciences, 94(5), 1079-1089. 